\title{
Development of a Detection System for Expressed Genes in Isolated Single Jurkat Cells
}

\author{
Shunsuke Furutani ${ }^{1,2}$, Naoki Shozen ${ }^{2}$, Hidenori Nagai ${ }^{1}$, \\ Yuri Aoyama² and Izumi Kubo ${ }^{2, *}$ \\ ${ }^{1}$ Health Research Institute, National Institute of Advanced Industrial Science and Technology, \\ 1-8-31 Midorigaoka, Ikeda, Osaka 563-8577, Japan \\ ${ }^{2}$ Department of Bioinformatics, Faculty of Engineering, Soka University, \\ 1-236 Tangi, Hachioji, Tokyo 192-8577, Japan
}

(Received January 15, 2014; accepted July 10, 2014)

Key words: gene expression, single cell, RT-PCR, Jurkat cell, GAPDH

A novel detection system for an expressed gene in single cells based on hot celldirect reverse transcription polymerase chain reaction (RT-PCR) using a device for cell isolation was developed. The system comprised a microscope to observe single cells and a thermal cycler to perform RT-PCR. We detected the mRNA of the glyceraldehyde3-phosphate dehydrogenase (GAPDH) gene in isolated Jurkat cells. In addition to the observation of single cells in microchambers after cell isolation, a marked fluorescence increase of the RT-PCR product from a single cell was observed with this new detection system. Relative fluorescence intensity (RFI), which was attributed to the level of GAPDH gene expression in each cell, was $2.30 \pm 0.41$ (ranging from 1.7 to 3.1) of the chambers with a single cell after RT-PCR, while that with no cells was $1.01 \pm 0.01$. The RFI of expressed $\beta$-actin was $2.63 \pm 0.70$ (ranging from 1.2 to 3.3 ) and similar to that of GAPDH. These results indicate that the gene expression level is different in each single cell, even when the expressed gene is a housekeeping gene, such as GAPDH and $\beta$-actin.

\section{Introduction}

Recently, the development of microfluidic devices for chemical and biochemical analyses has attracted major interest. Microfluidic devices integrate several laboratory processing steps into a single portable platform, in which miniaturization enables highly parallelized, multiplexed and fast detection processes. ${ }^{(1)}$ These systems, often referred to as lab-on-a-chip devices, typically contain several reaction chambers for simultaneously handling sample and reagent with actuation and detection mechanisms. The advantages of these systems include reduced reagent consumption, space requirements and analysis time. ${ }^{(2,3)}$ By exploiting these advantages, microfluidic devices are being increasingly

*Corresponding author: e-mail: kubo@soka.ac.jp 
used for research in the life sciences. In particular, the handling of a small amount of reagent has enabled the manipulation of a single cell on a microfluidic device. ${ }^{(4-7)}$

Centrifugally driven microfluidic platforms facilitate the precise handling of fluidic samples at the microscale. Liquid is driven by centrifugal pressure generated by spinning the disc at specific rotational frequencies. ${ }^{(8)}$ The advantage of this type of device is the elimination of bulky external pumps required to manipulate liquids. Only a low-power and low-cost rotational motor is required to spin the disc. These microfluidic platforms are generally referred to as "lab-on-a-disc" devices. ${ }^{(9)}$ It has been shown that flow rates from $5 \mathrm{~nL} \mathrm{~s}^{-1}$ to over $0.1 \mathrm{~mL} \mathrm{~s}^{-1}$ can be centrifugally generated. ${ }^{(10)}$

Since the polymerase chain reaction (PCR) was first described in $1985,{ }^{(11)}$ it has become an essential method in the field of genetic analysis, providing a method of amplifying DNA sequences of interest. A large variation in gene expression and behavior has been observed for genetically identical cells. ${ }^{(12,13)}$ Reverse transcription PCR (RT-PCR) is presently a gold standard to examine expressed mRNA. In the case of RT-PCR of cells in a tube, the difference in gene expression between single cells cannot be detected because the level of an expressed gene of all cells is averaged in the tube. By isolating a single cell from a large number of cells, an understanding of the behavior of that individual cell would be possible. A key to this process would be the detection of expressed mRNA in each cell. Recently, gene expression analysis for single cells on microdevices has gained considerable interest. ${ }^{(14-17)}$ However, it is necessary to add the reaction mixture for RT-PCR into the reaction chamber on these microdevices after cell isolation and lysis. Therefore, high-throughput detection for gene expression analysis in single cells is difficult on these microdevices because the system is very complicated. To detect a specific expressed gene among a large number of single cells, the microdevice needs to combine simple single cell isolation in a high-throughput manner and RT-PCR for single cells in the same reaction chamber.

We have developed an original compact disc (CD)-shaped device with microchannels and microchambers for single cell isolation, ${ }^{(18)}$ and succeeded in sequential PCR of the invA gene from Salmonella enterica cells isolated on a device fabricated from silicon and glass. ${ }^{(19)}$ We have also reported a method of performing cell lysis and RT-PCR of mRNA in the same chamber by heat treatment, which we named hot cell-direct RT-PCR, and the fluorescence detection of RT-PCR products was carried out using a double-dye probe. ${ }^{(20)}$ Hot cell-direct RT-PCR for single cells is important to investigate the expressed gene of each single cell. After hot cell-direct RT-PCR on the device, the fluorescence of each microchamber was detected using an image analyzer (LAS-3000, Fujifilm, Japan). The number of microchambers with cells estimated using a Poisson distribution was positively correlated to the number of chambers that showed an increase in fluorescence, which implied the presence of a cell or cells in the chamber. These results suggest that hot cell-direct RT-PCR in our device has the potential to detect mRNA in an isolated cell at the single cell level. However, whether a chamber contained a cell or not could not be determined, because the image of chambers using the image analyzer before and after the isolation of a single cell could not be precisely observed due to the limitations of the resolution of the image analyzer. Since the area of each pixel of the image analyzer is 50 $\times 50 \mu \mathrm{m}^{2}$, it is too large to obtain the image of a single cell $(10-20 \mu \mathrm{m})$. However, the 
image of U-shaped microchambers of the device, whose size is $300 \mu \mathrm{m}$ (width) $\times 200$ $\mu \mathrm{m}$ (height), cannot be obtained precisely. In fact, the pixels detected as microchambers included not only the microchamber itself but also the surrounding platform. Thus, the measured fluorescence of a microchamber was the average fluorescence of the chamber and the periphery. To detect accurate fluorescence, a detection system with a high resolution is necessary. The observation of single cells in microchambers under a microscope and the detection of the fluorescence intensity of each microchamber before and after hot cell-direct RT-PCR are definitely necessary to verify the amplification of mRNA in a single cell.

In this work, we developed a detection system that could acquire an image of all microchambers on the device automatically with a sufficiently high resolution to obtain the image of each cell and the shape of microchambers, which could not be obtained using the image analyzer, and could detect the amplification of mRNA in a single cell by hot cell-direct RT-PCR in each microchamber. This detection system for an expressed gene in a single cell comprised a microscope that could observe single cells in microchambers, a control system to acquire the image of all microchambers, and a thermal cycler to perform hot cell-direct RT-PCR on the device. Furthermore, more accurate and sensitive fluorescence detection was possible with this detection system. A marked increase in fluorescence of single cells in microchambers after RT-PCR of two housekeeping genes, glyceraldehyde-3-phosphate dehydrogenase (GAPDH) and $\beta$-actin, was automatically confirmed using this detection system, and the remarkable performance of the system was demonstrated when compared with that of the image analyzer.

\section{Materials and Methods}

\subsection{Reagents and sample preparation}

RT-PCR reagents and the preparation of Jurkat human leukemia T cells (DS Pharma Biomedical, Japan) have been described in detail, in ref. 20. In brief, Jurkat cells were cultured in RPMI 1640 medium (Gibco, Life Technologies, Japan) supplemented with $10 \%$ fetal bovine serum at $37{ }^{\circ} \mathrm{C}$ and $5 \% \mathrm{CO}_{2}$. After proliferation, the Jurkat cells were washed with $10 \mathrm{mmol} \mathrm{L}^{-1}$ phosphate buffer saline (pH 7.6) (PBS) three times at 1000 rpm for 3 min. RT-PCR was performed using Tth DNA polymerase (Roche Diagnostics $\mathrm{GmbH}$, Germany). RNase inhibitor $\left(40{\left.\mathrm{U} \mu \mathrm{L}^{-1}\right) \text { and dNTP mixture }(2.5 \mathrm{mmol} \mathrm{L}}^{-1}\right.$ each) were purchased from Ambion, Life Technologies, Japan. We used a double-dye probe and primer for GAPDH and $\beta$-actin (NIPPON EGT, Japan) to detect RT-PCR products by fluorescence. These probes were labeled with 6-carboxyfluorescein (FAM) and with a quencher. Segments of 86-bp and 63-bp for GAPDH and $\beta$-actin gene mRNA in Jurkat cells were amplified by RT-PCR. Jurkat cells were suspended in the reaction mixture that served both for isolation and RT-PCR. The Jurkat cells $\left(200\right.$ cells $\left.\mu \mathrm{L}^{-1}\right)$ were mixed

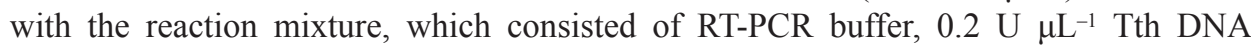
polymerase, $2.5 \mathrm{mmol} \mathrm{L}^{-1} \mathrm{Mn}(\mathrm{OAc})_{2}$ solution, $1.6 \mathrm{U}_{\mu} \mathrm{L}^{-1} \mathrm{RNase}$ inhibitor, $400 \mu \mathrm{mol} \mathrm{L}^{-1}$ dNTP mixture, $400 \mathrm{nmol} \mathrm{L}^{-1}$ forward and reverse primers, and $200 \mathrm{nmol} \mathrm{L}^{-1}$ probe for GAPDH or $\beta$-actin. 


\subsection{Fabrication of CD-shaped device}

The fabrication of the CD-shaped device was performed according to a method previously described in ref. 20. Briefly, the microchannel and microchambers (a depth of $40 \mu \mathrm{m})$ were fabricated by deep reactive ion etching (deep-RIE) on a silicon wafer $(\phi$ $=10 \mathrm{~cm}$, the thickness is $525 \mu \mathrm{m}$ ). The etched silicon wafer was anodically bonded to a glass plate (thickness of $500 \mu \mathrm{m})$ that has holes $(\phi=2 \mathrm{~mm})$ for inlets and vents bored by microblasting. Twenty-four microchannels are arranged on a device, and $313 \mathrm{U}$-shaped microchambers are arrayed on the outer side of each microchannel. The width of each channel is $100 \mu \mathrm{m}$. The dimensions of the microchambers are $300 \mu \mathrm{m}$ (width) $\times 200 \mu \mathrm{m}$ (height) $\times 40 \mu \mathrm{m}$ (depth), the gap between microchambers is approximately $200 \mu \mathrm{m}$, and the microchamber can accommodate approximately $1 \mathrm{~nL}$ of sample solution [Fig. 1(a)].

In this experiment, the hydrophobicity of each microchannel and microchambers was very important. Without a hydrophobic treatment, the RT-PCR reagents in the microchambers moved to the neighboring microchambers during RT-PCR. Therefore, to prevent the displacement of solution to neighboring microchambers, we modified the surface of the microchannels and microchambers to be highly hydrophobic by applying triethoxymethylsilane (Wako, Japan). For this modification, we infused 3 $\mu \mathrm{L}$ of triethoxymethylsilane into each microchannel by capillary force, followed by baking at $80{ }^{\circ} \mathrm{C}$ overnight. After baking, the triethoxymethylsilane did not remain in the microchannel and microchambers.

\subsection{Hot cell-direct RT-PCR in tube}

The hot cell-direct RT-PCR method has been described in detail, in ref. 20. In brief, hot cell-direct RT-PCR is a method for lysing cells and performing RT-PCR in the same reaction container such as a tube or a chamber. Tth DNA polymerase is a thermostable polymerase and has high reverse transcription activity. Therefore, it was possible to perform RT-PCR in the reaction mixture using Tth DNA polymerase after heat treatment for cell lysis at $95{ }^{\circ} \mathrm{C}$ for $10 \mathrm{~min}$. In this study, Tth DNA polymerase was used for both reverse transcription and PCR in the same reaction container. Jurkat cells were suspended in the reaction mixture for RT-PCR (1000 cells $\left.\mu \mathrm{L}^{-1}\right)$. After the cell lysis step, thermal cycling was initiated at $42{ }^{\circ} \mathrm{C}$ for $15 \mathrm{~min}$ as the RT step, followed by 40 PCR cycles of $94{ }^{\circ} \mathrm{C}$ for $30 \mathrm{~s}$ (denaturation) and $60{ }^{\circ} \mathrm{C}$ for $60 \mathrm{~s}$ (annealing and extension) using a 7500 real-time PCR system (Applied Biosystems, Japan). The fluorescence intensity of RT-PCR products of the GAPDH gene was measured using the real-time PCR system.

\subsection{Detection system for expressed gene in single cells}

We designed a system to acquire the images of a single cell before its lysis and to detect the fluorescence image of the microchambers before and after hot cell-direct RT-PCR of the target gene in this study. The system comprised an epifluorescence microscope (Olympus, Japan), heating-cooling equipment as a thermal cycler (UT70U100F, Ampere, Japan), a controllable XY stage (Ohkura Industry, Japan), a CCD camera (Clara, Andor, Northern Island) and a personal computer for system controls [Fig. 1(b)]. This system was provided by Ohkura Industry, Japan. The single cells or RT-PCR products 


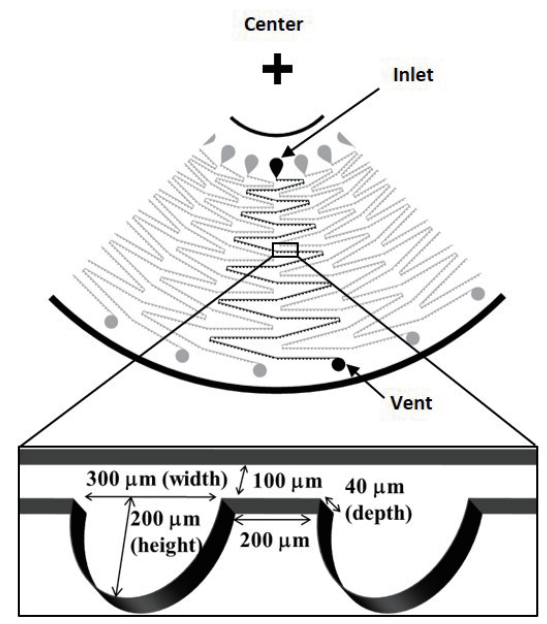

(a)

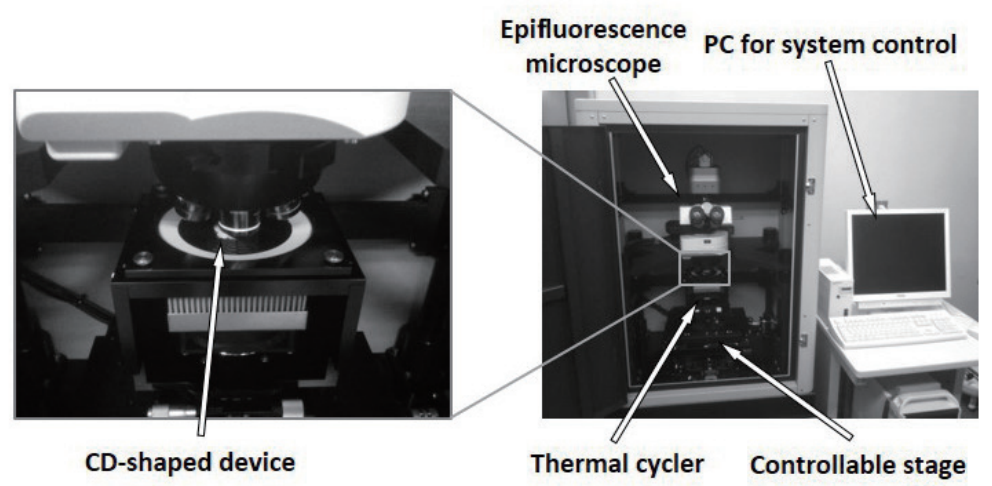

(b)

Fig. 1. Microfluidic design of a CD-shaped device and detection system for an expressed gene in single cells. (a) Microfluidic design and details of microchannel and microchambers of device. (b) The detection system was composed of an epifluorescence microscope, a thermal cycler, a controllable stage and a personal computer for system control. The device was set on the stage of the detection system.

in microchambers were observed with an epifluorescence microscope. The images of all microchambers were automatically acquired with the movement of the controllable $\mathrm{XY}$ stage along the microchannels from inlet to outlet. In this detection system, to prevent defocusing by acquiring the image of all microchambers, alignment of two anchoring points on the device was carried out prior to the imaging of microchambers. Furthermore, the fluorescence intensities of each microchamber were quantified from 
the fluorescence images. The personal computer of the system controls the temperature of the thermal cycler, the movement of the device on the controllable XY stage, and the conditions of the exposure time for the fluorescence detection.

\subsection{Single cell isolation on CD-shaped device}

Prior to RT-PCR of the mRNA in Jurkat cells on the device, single cells were isolated on the device. One microliter of Jurkat cell suspension $\left(200\right.$ cells $\left.\mu \mathrm{L}^{-1}\right)$ was introduced into an inlet of the device. The device was then rotated clockwise at $3500 \mathrm{rpm}$ for 30 s using a spin-coater (MS-A100, Mikasa, Japan). The cell suspension flowed through the microchambers [Fig. 2(b)] and cells were isolated into individual microchambers in accordance with a Poisson distribution [Fig. 2(c)]. Sequentially, excess solution was finally drained from each microchannel at $3500 \mathrm{rpm}$. After spinning the device, the reaction mixture was completely isolated in the microchambers.

\subsection{Hot cell-direct RT-PCR on CD-shaped device}

Hot cell-direct RT-PCR of single Jurkat cells on the device, which has been reported in ref. 20, was slightly modified in this study. After single cell isolation, the device was placed on the stage of a conventional thermal cycler (Astec, Japan) or the detection system. These stages were shaped to fit the device. Thermal cycling was initiated at $95{ }^{\circ} \mathrm{C}$ for $10 \mathrm{~min}$ as heat treatment for cell lysis and $42{ }^{\circ} \mathrm{C}$ for $15 \mathrm{~min}$ as the RT step, followed by $40 \mathrm{PCR}$ cycles of $94{ }^{\circ} \mathrm{C}$ for $20 \mathrm{~s}$ (denaturation), $54{ }^{\circ} \mathrm{C}$ for $20 \mathrm{~s}$ (annealing) and $72{ }^{\circ} \mathrm{C}$ for $20 \mathrm{~s}$ (extension), and a further $72{ }^{\circ} \mathrm{C}$ for $5 \mathrm{~min}$ as the final extension step.

(a)

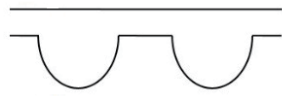

(b)

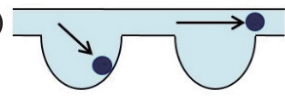

(c)

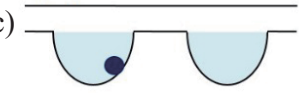

(d)

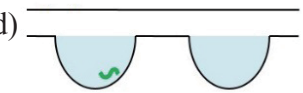

(e)

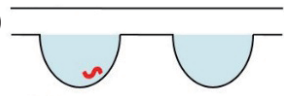

(f)

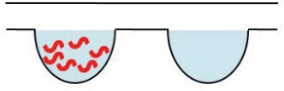

(g)

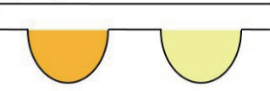

- cell $\sim$ mRNA

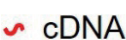

Fig. 2. A scheme for single cell isolation and hot cell-direct RT-PCR on device. (a) A large number of microchambers align along a microchannel. (b) The cells flow through the microchannel and the microchambers. (c) The cells are distributed into individual microchambers after flowing through the applied cell suspension. (d) The cells are lysed at $95^{\circ} \mathrm{C}$ for $10 \mathrm{~min}$. (e) The mRNA is reverse transcribed to cDNA. (f) The cDNA is amplified by PCR. (g) An increase in fluorescence of the double dye probe for RT-PCR products is measured using the detection system. 
A scheme of the hot cell-direct RT-PCR reaction for a single cell in the microchamber is indicated in Fig. 2. The fluorescence intensities of RT-PCR products of the GAPDH and $\beta$-actin genes were measured using the detection system before and after hot cell-direct RT-PCR on the device. The relative fluorescence intensity (RFI) indicated the after-tobefore ratio of hot cell-direct RT-PCR.

\section{Results and Discussion}

\subsection{Detection of fluorescence of hot cell-direct RT-PCR products}

Before measuring the fluorescence intensity after hot cell-direct RT-PCR of single cells in the device, the fluorescence of the RT-PCR products of the expressed GAPDH gene in a tube was detected using this detection system. The concentration of Jurkat cells in a tube was set at 1000 cells $\mu \mathrm{L}^{-1}$, which corresponded to a density of one cell per microchamber. Figure 3(a) indicates the increase in fluorescence of the samples by hot cell-direct RT-PCR in a tube using the real-time PCR system. As for the reaction mixture that contained 1000 Jurkat cells $\mu \mathrm{L}^{-1}$, the increase in fluorescence was clearly confirmed and saturated after 30 PCR cycles. In contrast, the fluorescence intensity of the negative control containing no cells did not increase at all.

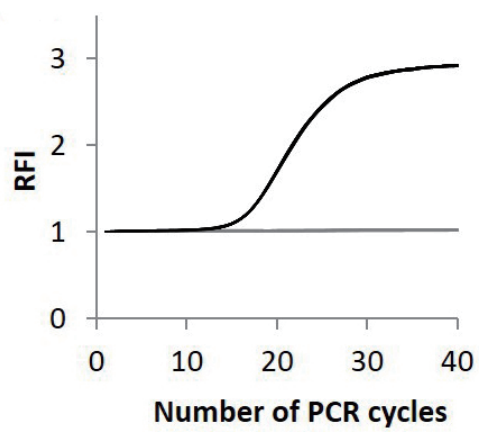

(a)

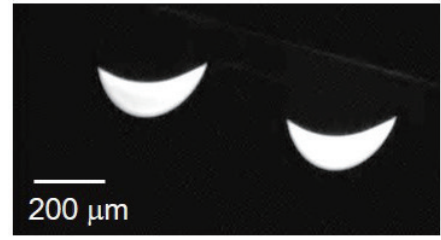

(b)

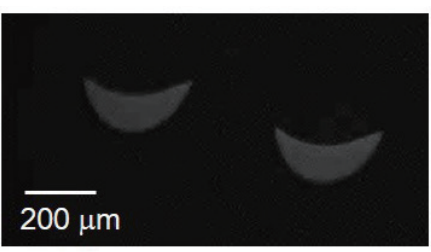

(c)

Fig. 3. Hot cell-direct RT-PCR in a tube using the real-time PCR system and fluorescence images obtained with the detection system. (a) The black line indicates the positive control with Jurkat cells $\left(1000\right.$ cells $\left.\mu \mathrm{L}^{-1}\right)$. The gray line indicates the negative control without Jurkat cells. (b) Fluorescence image of RT-PCR products of expressed GAPDH gene (1000 Jurkat cells $\mu \mathrm{L}^{-1}$ ). (c) Fluorescence image of negative control without Jurkat cells after hot cell-direct RT-PCR. 
To examine the fluorescence of RT-PCR products of the GAPDH gene using our detection system, RT-PCR products obtained using the real-time PCR system were introduced and completely separated to each microchamber in the device as positive and negative controls. Figures 3(b) and 3(c) indicate the fluorescence images of the microchambers with positive and negative controls, respectively, using the detection system after hot cell-direct RT-PCR in a tube. The RFIs measured using the detection system, the after-to-before ratio of hot cell-direct RT-PCR, of the positive and negative controls were $2.92 \pm 0.01$ and $1.02 \pm 0.01$, respectively $(n=3)$. Thus, the detection system could be used to measure the increase in fluorescence of RT-PCR products. Furthermore, the small standard deviation (SD) of RFI implied that the amounts of solution in each microchamber were almost equal. When we examined the positive and negative controls on the device and measured the fluorescence signal using the image analyzer, the RFIs of the positive and negative controls were found to be $1.34 \pm 0.08$ and $1.04 \pm 0.04$, respectively. The RFI of the positive control using the image analyzer was lower than that using the detection system, while that of the negative control using the image analyzer and that using the detection system were almost the same. The SDs of the positive and negative controls were larger with the image analyzer than with the detection system. It was considered that the fluorescence observed with the image analyzer was not necessarily from the microchamber itself because the pixel size was not small enough to cover the chamber precisely. The observed fluorescence included the surrounding platform, which in fact corresponds to a background signal, and the ratio of the area of the surrounding platform signal to the total area of the fluorescence signal of each chamber was different from chamber to chamber. Thus, the observed fluorescence was less accurate than that using the detection system, and SD might have become larger. These results imply that measurements of fluorescence in the microchamber with the image analyzer were not accurate. In contrast, the new detection system was accurate.

\subsection{Single cell hot cell-direct RT-PCR in CD-shaped device}

To examine the performance of the new detection system, the fluorescence intensity of the device after hot cell-direct RT-PCR for GAPDH was first measured using the image analyzer. A conventional thermal cycler was used for RT-PCR of the device after the isolation of Jurkat cells $\left(200\right.$ cells $\left.\mu \mathrm{L}^{-1}\right)$. Although which microchamber entraps a single cell from the image obtained using the image analyzer before RT-PCR cannot be determined, the RFI of the microchamber with a single cell should be higher than that of a microchamber without a cell after RT-PCR. The RFI of microchambers without any cells remained constant after RT-PCR. The fluorescence intensities of microchambers measured using the image analyzer are shown in Fig. 4(a). We observed that RFI of most chambers was about 1.0 but some chambers showed a higher RFI. As already mentioned, the image of a Jurkat cell could not be obtained when the image analyzer was used because of its low resolution, and the microchamber showing a higher RFI was recognized as being the chamber entrapping a Jurkat cell. As for RFIs obtained using the image analyzer, the microchambers showed RFIs higher than 1.21 and were considered to have a single cell while those with an RFI lower than 1.21 contained no cell because the maximum RFI of the negative control was 1.21. Statistically, the 

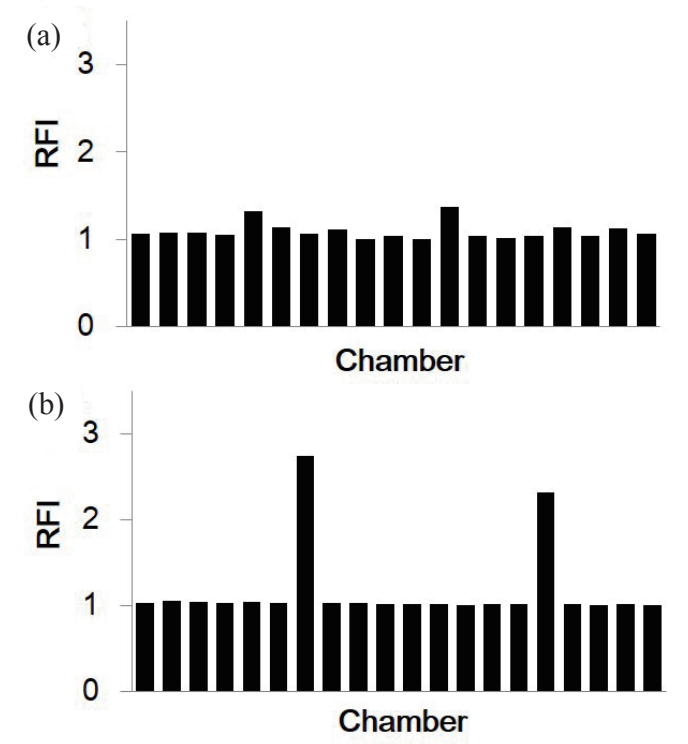

Fig. 4. Typical RFIs following the detection of fluorescence using the image analyzer and detection system. RFIs for GAPDH of each microchamber are shown on the same microchannel using (a) image analyzer or (b) detection system. These chambers are all adjacent on the CD.

number of microchambers showing higher fluorescence after RT-PCR corresponded to the value evaluated using a Poisson distribution, according to our previous study in ref. 21. The fluorescence intensities of microchambers were also measured using the new detection system [Fig. 4(b)], which could acquire images of all the microchambers in a microchannel. Then, the discrimination of microchambers containing single Jurkat cells from all microchambers was possible using this new detection system. Hot cell-direct RT-PCR was performed with the thermal cycler equipped in the detection system for real-time detection of RFI, which could be measured. High RFIs (2.74 and 2.42) were easily identified for the two microchambers, which corresponded to chambers containing a single cell.

In the case of the image analyzer, the image of a single cell could not be observed as mentioned above. On the other hand, the fluorescence image before hot cell-direct RT-PCR for GAPDH using the system, indicating a single Jurkat cell trapped in the microchamber, was obtained [Fig. 5(a)]. Although the size of the cell was $10 \mu \mathrm{m}$ in diameter and rather small compared with the microchamber, it was clearly observed in the right chamber in Fig. 5(a). Whether a chamber contained a cell or not could be clearly determined using the system. In the case of 200 Jurkat cells $\mu \mathrm{L}^{-1}$, approximately $10 \%$ of the observed microchambers had single cells, while a few microchambers had two or more cells. In this study, the expressed gene could be detected in single cells after the number of cells in microchambers was counted directly. Figure $5(\mathrm{~b})$ indicates 


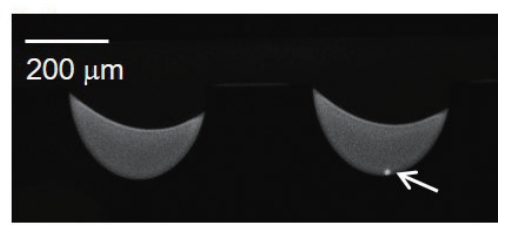

(a)

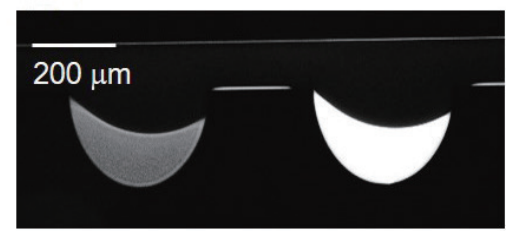

(b)

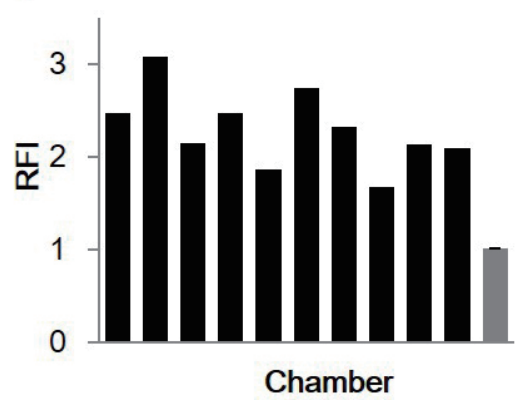

(c)

Fig. 5. Hot cell-direct RT-PCR for GAPDH in microchambers using the thermal cycler of the detection system. (a) Fluorescence image before hot cell-direct RT-PCR. Arrow indicates a single Jurkat cell in a microchamber. (b) Fluorescence image after hot cell-direct RT-PCR. (c) RFI in microchambers with a single Jurkat cell (black bars) or average RFI of ten microchambers with no cells (gray bar).

a fluorescence image of the same microchamber after hot cell-direct RT-PCR. Although the fluorescence intensity of the microchamber with no cells shows little increase, that of the microchamber with a single Jurkat cell clearly increased. Figure 5(c) indicates the RFI after hot cell-direct RT-PCR in microchambers with a single Jurkat cell and with no cells. The RFIs for GAPDH of ten microchambers with a single Jurkat cell ranged from 1.7 to $3.1(2.30 \pm 0.41)$. In contrast, the fluorescence intensities of ten microchambers with no cells showed little increase after hot cell-direct RT-PCR. The RFIs of microchambers with two cells ranged from 3.2 to 3.6, since the fluorescence was saturated by 40 PCR cycles. To verify this phenomenon, the expression of another housekeeping gene, $\beta$-actin, in single cells was examined using the new detection system. The RFIs for $\beta$-actin of ten microchambers with a single Jurkat cell ranged from 1.2 to $3.3(2.63 \pm$ 0.70 ), whereas the fluorescence of microchambers with no cells showed little increase, a similar pattern as observed for GAPDH. This result indicates the possibility that the gene expression level was different in each cell, even when the expressed gene was a housekeeping gene, such as GAPDH and $\beta$-actin. Besides us, several researchers also reported the gene expression heterogeneity at the single cell level within genetically identical cell populations in refs. 22 and 23. This gene expression heterogeneity was 
considered to be caused by the different cell types and different cell cycle phases. Therefore, our results of gene expression heterogeneity would also be caused by the different cell cycle phases.

To examine the performance of a thermal cycler in the new detection system, hot cell-direct RT-PCR was performed using a conventional thermal cycler as well as the detection system. These fluorescence intensities of GAPDH RT-PCR products were measured using the detection system. The fluorescence intensities of microchambers with a single Jurkat cell and with no cells after hot cell-direct RT-PCR using the conventional thermal cycler were almost the same as the RFIs using the detection system. The averaged RFIs and SDs obtained for these GAPDH measurements are listed in Table 1. The RFIs of the microchambers with single cells as assessed using the image analyzer were 1.25 times higher than those with no cells but two times higher when the new detection system was used. The SD for RFIs of the microchamber containing a single or no cell may have arisen because the difference in expression level for each single cell could not be confirmed using the image analyzer. Consequently, the SD for RFIs of the microchamber with cells as assessed using the image analyzer was comparable to the SD for RFIs of the negative control. On the other hand, the fluorescence intensity was measured accurately using the detection system because the SD for RFIs of the microchamber with no cells was very small and remained constant. The RFIs of the microchambers with single cells measured using the detection system were higher than those using the image analyzer. Furthermore, the RFIs and SDs after hot celldirect RT-PCR using the thermal cycler of the detection system were almost the same as those using the conventional thermal cycler. This implies that there is no difference in fluorescence after RT-PCR between the detection system and the thermal cycler. The SD for RFIs of the no-cell control as assessed using the new detection system was so small that the measured RFIs were considered to be accurate. Thus, RFI truly reflects a difference in the amount of RT-PCR product. The SDs for the RFIs of microchambers with a single cell were significantly high and could indicate a difference in the expression level for each single cell. Only the new detection system could accurately detect fluorescence in cells following RT-PCR.

Table 1

Performance of fluorescence detection and thermal cycler of new detection system.

\begin{tabular}{lllcc}
\hline \multirow{2}{*}{ Fluorescence detection } & \multirow{2}{*}{ Thermal cycling } & Number of cells & \multicolumn{2}{c}{ RFI } \\
\cline { 4 - 5 } & & & Average & SD \\
\hline \multirow{2}{*}{ Image analyzer } & Conventional & Single cell & \\
& thermal cycler & No cells* & 1.33 & 0.04 \\
\multirow{2}{*}{ Detection system } & Conventional & Single cell & 2.44 & 0.42 \\
& thermal cycler & No cells & 1.06 & 0.01 \\
\multirow{2}{*}{ Detection system } & \multirow{2}{*}{ Detection system } & Single cell & 2.30 & 0.41 \\
& & No cells & 1.01 & 0.01 \\
\hline
\end{tabular}

"It was not confirmed accurately using the image analyzer. 
The quantitative determination of an expressed gene has been performed by separately detecting RT-PCR products for a housekeeping gene and a target gene, although it would be theoretically possible to determine the expression of both genes simultaneously using two probes and primers specific for each gene. Furthermore, when the fluorescence intensity is measured after each PCR cycle using the detection system, the quantitative determination of the expressed gene would be possible at the single cell level. RT-PCR for single cells on this device is expected to have great potential for detecting different cell states of many single cells such as developing embryos, embryonic stem cells or induced pluripotent stem (iPS) cells, because not only Jurkat cells but also fibroblast cells have been isolated in the microchambers of this device in ref. 21.

\section{Conclusions}

A novel system to detect gene expression in isolated single Jurkat cells was developed. This system was composed of an epifluorescence microscope, a thermal cycler, a controllable stage and a personal computer for system control. Jurkat cells were isolated in the microchambers stochastically on the device and observed using this detection system. The confirmation of the number of cells in each microchamber could be realized first using the detection system before RT-PCR but it could not be done using the image analyzer. The RFIs for the microchambers with single cell using the detection system was $2.30 \pm 0.41$ and RFIs for microchamber with cell or cells obtained by the image analyzer was $1.33 \pm 0.04$. The RFIs obtained using the detection system were more accurate and higher than those assessed using the image analyzer. The SD of the detection system would indicate a difference in the expression level for each single cell because of its accuracy. In conclusion, single cells could be observed, RT-PCR could be performed, and the fluorescence and gene expression level could be detected with the new detection system but not using the image analyzer. The gene expression level differed in each single cell even when one of the expressed genes was a housekeeping gene.

\section{Acknowledgements}

This work was supported in part by a Grant-in-Aid for Scientific Research (C) from the Ministry of Education, Culture, Sports, Science and Technology (MEXT), Japan, and in part by funding from MEXT, the Matching Fund for Private Universities, S1001013, 2010-2015.

\section{References}

1 S. J. Trietsch, T. Hankemeier and H. J. van der Linden: Chemom. Intell. Lab. Syst. 108 (2011) 64.

2 G. M. Whitesides: Nature 442 (2006) 368.

3 P. S. Dittrich, K. Tachikawa and A. Manz: Anal. Chem. 78 (2006) 3887. 
4 W.-H. Tan and S. Takeuchi: Lab Chip 8 (2008) 259.

5 L.-S. Jang and M.-H. Wang: Biomed. Microdevices 9 (2007) 737.

6 D. D. Carlo, N. Aghdam and L. P. Lee: Anal. Chem. 78 (2006) 4925.

7 Y. Tanaka, K. Sato, T. Shimizu, M. Yamato, T. Okano and T. Kitamori: Biosens. Bioelectron. 23 (2007) 449.

8 M. J. Madou, L. J. Lee, S. Daunert, S. Lai and C. H. Shih: Biomed. Microdevices 3 (2001) 245.

9 M. Madou, J. Zoval, G. Jia, H Kido, J. Kim and N. Kim: Annu. Rev. Biomed. Eng. 8 (2006) 601.

10 D. Duffy, H. Gillis, J. Lin, N. Sheppard and G. Kellogg: Anal. Chem. 71 (1999) 4669.

11 R. K. Saiki, S. Scharf, F. Faloona, K. B. Mullis, G. T. Horn, H. A. Erlich and N. Arnheim: Science 230 (1985) 1350.

12 C. V. Rao, D. M. Wolf and A. P. Arkin: Nature 420 (2002) 231.

13 K. Kuang, I. Biran and D. Walt: Anal. Chem. 76 (2004) 6282.

14 J. S. Marcus, W. F. Anderson and S. R. Quake: Anal. Chem. 78 (2006) 3084.

15 J. S. Marcus, W. F. Anderson and S. R. Quake: Anal. Chem. 78 (2006) 956.

16 N. M. Toriello, E. S. Douglas, N. Thaitrong, S. C. Hsiao, M. B. Francis, C. R. Bertozzi and R. A. Mathies: PNAS 105 (2008) 20173.

17 Y. Marcy, T. Ishoey, R. S. Lasken, T. B. Stockwell, B. P. Walenz, A. L. Halpern, K. Y. Beeson, S. M. Goldberg and S. R. Quake: PLoS Genet. 3 (2007) 1702.

18 S. Furutani, H. Nagai and I. Kubo: Sensor Lett. 6 (2008) 961.

19 S. Furutani, H. Nagai, Y. Takamura and I. Kubo: Anal. Bioanal. Chem. 398 (2010) 2997.

20 S. Furutani, H. Nagai, Y. Takamura, Y. Aoyama and I. Kubo: Analyst 137 (2012) 2951.

21 I. Kubo, S. Furutani and H. Nagai: ECS Trans. 16 (2008) 1.

22 Y. Taniguchi, P. J. Choi, G.-W. Li, H. Chen, M. Babu, J. Hearn, A. Emili and X. S. Xie: Science 329 (2010) 533.

23 Y. Sasagawa, I. Nikaido, T. Hayashi, H. Danno, K. D. Uno, T. Imai and H. R. Ueda: Genome Biol. 14 (2013) R31. 